\title{
Pathological Gambling Induced by Dopamine Antagonists: A Case Report
}

\author{
Philipp Grötsch • Claudia Lange • Gerhard A. Wiesbeck • \\ Undine Lang
}

Published online: 20 December 2013

(C) Springer Science+Business Media New York 2013

\begin{abstract}
Pathological gambling is defined as inappropriate, persistent, and maladaptive gambling behaviour. It is a non-pharmacological addiction classified as an impulse control disorder. However, pathological gambling has been associated with dopamine agonist use. Here we report of a 28-year-old man with a first major depressive episode and a post-traumatic stress disorder who has been treated with a combination of the serotonine/noradrenaline reuptake inhibitor duloxetine and the tricyclic antidepressant maprotiline. The administration of antipsychotic flupentixole (up to $7 \mathrm{mg}$ ) turned this slight online poker gambler into an excessive gambler. Only after the discontinuation of the antidopaminergic agents and the switch to bupropion did this gambling behaviour stop which suggests a causal relationship between dopamine antagonists and pathological gambling.
\end{abstract}

Keywords Antipsychotic agents · Gambling behaviour - Dopamine antagonists

\section{Background}

Pathological gambling is defined by the DSM-IV criteria as inappropriate, persistent, and maladaptive gambling behaviour that has repercussions on family, personal and professional life. It is classified as an impulse control disorder and is primarily understood as a non-pharmacological addiction. However, pathological gambling has also been reported as a complication of neurodegenerative disorders, particularly Parkinson's disease, and is a common side effect of treatment with dopamine agonists (Seedat et al. 2000).

P. Grötsch · C. Lange $(\bowtie) \cdot$ G. A. Wiesbeck $\cdot$ U. Lang

Psychiatric Hospital of the University of Basel, Wilhelm Klein-Strasse 27, 4012 Basel, Switzerland

e-mail: claudia.lange@upkbs.ch 


\section{Case Report}

We report here the case of a 28 -year-old male with a first major depressive episode (MDD) with atypical clinical presentation (according to DSM-IV) and a post-traumatic stress disorder (PTSD) who has been treated with a combination of the serotonine/noradrenaline reuptake inhibitor duloxetine and the tricyclic antidepressant maprotiline. According to DSM-IV the depression showed an atypical course, featuring mood reactivity, hypersomnia, the feeling of being paralyzed and an increased sensitivity to rejection.

Because of rumination due to MDD and PTSD, the antipsychotic flupentixole (up to $7 \mathrm{mg}$ ) was administered, which turned a slight online poker gambling within several days into an excessive gambling behaviour with total loss of self-control. There was no previous history of problem gambling or other addictions neither in this individual nor in his family. Soon, the patient spent up to 20,000 CHF within 1 week. Even though restrictions on access to money and internet were made in accordance with the patient the behaviour continued resulting in extreme losses within a short time, even by disposing valuables of his parents. While flupentixole was replaced by several D2 antagonists (olanzapine up to $7.5 \mathrm{mg}$, thioridazine up to $50 \mathrm{mg}$, sulpiride up to $200 \mathrm{mg}$ ) the intense craving for gambling lasted for several months. Discontinuation of the antidopaminergic agents and consecutive treatment with bupropion not only stopped the gambling behaviour but also the craving completely within several days. By now, after a follow-up of 18 months, the gambling behaviour has not recurred.

The temporal coincidence between the administration of the antipsychotic agents on the one hand and the gambling behaviour-particular the craving — on the other hand was striking in this case, strongly suggesting a causal relationship, from our point of view.

\section{Conclusion}

The D2 receptor has been implicated as a critical substrate in the modulation of psychostimulant reward (Volkow et al. 2001), and the D2 receptor has been implicated moreover in the genetic risk of pathological gambling (PG) (Lim et al. 2012). Also, neuroimaging studies have consistently shown deficits in D2 receptor binding in individuals who manifest cocaine or metamphetamine abuse (Nader and Czoty 2005). Whereas the partial dopamine agonist aripiprazole was found to induce PG in three cases (Smith et al. 2011) and is in line with the well-known fact that dopamine agonists can provoke PG (Miura et al. 2009), we report here the first clinical case of PG induced by dopamine antagonists, to our knowledge.

However there are some previous observations suggesting a reciprocal effect between dopamine action at the D2-receptor and gambling behaviour: Haloperidol can augment the pleasurable effects of the slot machine game in pathological gamblers as reflected by enjoyment, excitement and involvement scales (Zack and Poulos 2009). In this study Haldol had no effect on the pregame desire to gamble but Haldol amplified the priming effect of the slot machine in pathological gamblers. It also enhanced the salience of gambling words relative to neutral words. In a series of studies Volkow et al. (2001) showed that the lower the availability of dopaminergic D2 receptors, the greater was the drug craving.

Our observation is also in line with a double-blind placebo-controlled study showing that modafinil, an agent increasing the synaptic dopamine concentration, reduced the 
reinforcing effects of slot machine gambling in pathological gambling subjects, at least if these patients were highly impulsive (Zack and Poulos 2007).

While the clinical course of the depression in this patient was stable during onset and cessation of the PG behaviour, the fact that it showed atypical features, i.e. mood reacitivity, may have facilitated the onset of PG. Whether the atypical nature of the depression could also be responsible for the differing response of the PG behaviour to dopamine modulating agents, remains to be elucidated.

Conflict of interest None.

\section{References}

Lim, S., Ha, J., Choi, S. W., Kang, S. G., \& Shin, Y. C. (2012). Association study on pathological gambling and polymorphisms of dopamine $\mathrm{d} 1, \mathrm{~d} 2, \mathrm{~d} 3$, and $\mathrm{d} 4$ receptor genes in a Korean population. Journal of Gambling Studies, 28, 481-491.

Miura, J., Kikuchi, A., Fujii, A., Tateishi, T., \& Kaneko, S. (2009). Pathological gambling associated with cabergoline in a case of recurrent depression. Drug Discoveries \& Therapeutics, 3(4), 190-192.

Nader, M. A., \& Czoty, P. W. (2005). PET imaging of dopamine D2 receptors in monkey models of cocaine abuse: genetic predisposition versus environmental modulation. American Journal of Psychiatry, 162, 1473-1482.

Seedat, S., Kesler, S., Niehaus, D. J., \& Stein, D. J. (2000). Pathological gambling behaviour: emergence secondary to treatment of Parkinson's disease with dopaminergic agents. Depression and Anxiety, 11(4), 185-186.

Smith, N., Kitchenham, N., \& Bowden-Jones, H. (2011). Pathological gambling and the treatment of psychosis with aripiprazole: Case reports. British Journal of Psychiatry, 199, 158-159.

Volkow, N. D., Chang, L., Wang, G. J., Fowler, J. S., Ding, Y. S., Sedler, M., et al. (2001). Low level of brain dopamine D2 receptors in methamphetamine abusers: Association with metabolism in the orbitofrontal cortex. American Journal of Psychiatry, 158, 2015-2021.

Zack, M., \& Poulos, C. X. (2007). A D2 antagonist enhances the rewarding and priming effects of a gambling episode in pathological gamblers. Neuropsychopharmacology, 32, 1678-1686.

Zack, M., \& Poulos, C. X. (2009). Effects of the atypical stimulant modafinil on a brief gambling episode in pathological gamblers with high vs. low impulsivity. Journal of Psychopharmacology, 23, 660-671. 